\title{
Machine Perfusion versus Cold Storage for the Preservation of Kidneys Donated
}

\section{after Cardiac Death: a Multicenter Randomized Controlled Trial}

\section{Authors:}

Ina Jochmans, M.D. (1)

Cyril Moers, M.D. (2)

Jacqueline M. Smits, M.D., Ph.D. (3)

Henri G. D. Leuvenink, Ph.D. (2)

Jürgen Treckmann, M.D. (4)

Andreas Paul, M.D. (4)

Axel Rahmel, M.D. (3)

Jean-Paul Squifflet, M.D., Ph.D. (5)

Ernest van Heurn, M.D., Ph.D. (6)

Diethard Monbaliu, M.D., Ph.D. (1)

Rutger J. Ploeg, M.D., Ph.D. (2)

Jacques Pirenne, M.D., Ph.D. (1)

\section{Affiliations:}

(1) from the Department of Abdominal Transplant Surgery, University Hospitals Leuven, Catholic University Leuven, Leuven, Belgium

(2) from the Department of Surgery, University Medical Center Groningen, University of Groningen, Groningen, the Netherlands

(3) from Eurotransplant International foundation, Leiden, the Netherlands

(4) from the Department of General, Visceral, and Transplantation Surgery, University Hospital Essen, Essen, Germany

(5) from the Department of Abdominal Surgery and Transplantation, Centre Hospitalier Universitaire Sart Tilman, University of Liège, Liège, Belgium 
(6) from the Department of Surgery, University Hospital Maastricht, Maastricht, the Netherlands

\section{Correspondence:}

Ina Jochmans

University Hospitals Leuven

Department of Abdominal Transplant Surgery

Herestraat 49

B-3000 Leuven

Belgium

E-mail: ina.jochmans@med.kuleuven.be

Phone: +32-16-348727

Fax: $+32-16-348743$

\section{Sources of support and Conflicts of interest}

Organ Recovery Systems supported this trial.

IJ is a doctoral fellow associated with the Research Foundation-Flanders, Belgium.

$\mathrm{CM}$ receives grant support from the Dutch Kidney Foundation.

JP and DM received grant support from the government of Flanders, Belgium, in cooperation with

Organ Recovery Systems to study machine perfusion of liver grafts, for which they receive no salary.

$\mathrm{CM}, \mathrm{AP}, \mathrm{HGDL}$, JPS, and JP received congress travel grants from Organ Recovery Systems.

RJP receives consultancy fees from Bristol-Myers Squibb and grant support from the Dutch Kidney Foundation and the Eurotrans-Bio pro-donor project.

RJP and HGDL are listed as inventors in a patent on a portable preservation apparatus for donor organs.

Running Title: Kidney preservation in cardiac dead donors

This is the version of the paper as accepted by "Ann Surg".

This version has been through peer-review but still contains the authors' original formatting. It has not yet undergone the publisher's copy-editing and typesetting process, which will usually result in changes to the font and text alignment. 


\section{Mini-abstract}

In a multicenter randomized controlled trial hypothermic machine perfusion of kidneys donated after cardiac death was compared to the current standard of static cold storage. Hypothermic machine perfusion proved to be superior. It was associated with a reduced risk of delayed graft function and better early graft function.

\section{Abstract \\ Objective}

Hypothermic machine perfusion may improve outcome after transplantation of kidneys donated after cardiac death (DCD), but no sufficiently powered prospective studies have been reported. As organ shortage has led to an increased use of DCD kidneys, we aimed to compare hypothermic machine perfusion with the current standard of static cold storage preservation.

\section{Methods}

Eighty-two kidney pairs from consecutive, controlled DCD donors aged 16 years or older were included in this randomized controlled trial in Eurotransplant. One kidney was randomly assigned to machine perfusion and the contralateral kidney to static cold storage according to computer generated lists created by the permuted block method. Kidneys were allocated according to standard rules, with concealment of the preservation method. Primary endpoint was delayed graft function (DGF), defined as dialysis requirement in the first week after transplantation. All 164 recipients were followed until one year after transplantation.

Trial registration number: ISRCTN83876362

\section{$\underline{\text { Results }}$}

Machine perfusion reduced the incidence of DGF from $69.5 \%$ to $53.7 \%$ (adjusted odds ratio $0.43 ; 95 \%$ confidence interval $0.20-0.89 ; p=0.025)$. DGF was four days shorter in recipients of machine-perfused kidneys ( $p=0.082$ ). Machine-perfused kidneys had a higher creatinine clearance up to one month after transplantation $(p=0.027)$. One-year graft and patient survival was similar in both groups $(93.9 \%$ vs. $95.1 \%)$.

This is the version of the paper as accepted by "Ann Surg". This version has been through peer-review but still contains the authors' original formatting. It has not yet undergone the publisher's copy-editing and typesetting process, which will usually result in changes to the font and text alignment. 


\section{$\underline{\text { Conclusions }}$}

Hypothermic machine perfusion was associated with a reduced risk of DGF and better early graft function up to one month after transplantation. Routine preservation of DCD kidneys by hypothermic machine perfusion is therefore advisable.

This is the version of the paper as accepted by "Ann Surg".

This version has been through peer-review but still contains the authors' original formatting. It has not yet undergone the publisher's copy-editing and typesetting process, which will usually result in changes to the font and text alignment. 
Introduction

Kidney grafts can be preserved by either static cold storage or hypothermic machine perfusion. Static cold storage preserves grafts on melting ice after a cold vascular flush with a preservation solution. Hypothermic machine perfusion preserves the graft by continuous or pulsatile administration of a recirculating cold preservation solution $\left(1-10^{\circ} \mathrm{C}\right)$. Optimal preservation of kidney grafts is essential to reduce the risk of delayed graft function (DGF) after transplantation. ${ }^{1}$ Indeed, DGF negatively influences long-term graft survival, is associated with a higher risk of acute rejection, and causes increased mortality in older recipients. ${ }^{1-4}$ DGF inevitably augments postoperative costs due to prolonged hospital stay, the need for dialysis, and additional diagnostic procedures. ${ }^{5,6}$

Currently, due to the persistent donor shortage, kidneys donated after cardiac death (DCD) have become an important additional source of renal allografts in many countries. ${ }^{8}$ They have the potential to increase the number of kidney transplantations up to 4.5 times. ${ }^{7,8}$ Compared to kidneys donated after brain death (DBD), DCD kidneys suffer from a substantially higher incidence of DGF (28-88\% vs. $13-35 \%)$, which seriously limits their use. ${ }^{8-10}$ This increased rate of DGF is caused by inevitable exposure of these kidneys to renal warm ischemic injury during the period of circulatory arrest. Therefore, optimal preservation of DCD kidneys is crucial to reduce their intrinsically increased risk of DGF and allow a safer and wider use of this potentially large donor source.

Previous studies have suggested that machine perfusion of DCD kidneys results in better early function and improved graft survival compared to static cold storage. ${ }^{11-13}$ Other studies do not support this conclusion, however, and a comprehensive meta-analysis failed to show a statistically significant risk reduction of DGF in machine-perfused versus static cold-stored DCD kidneys. ${ }^{14-16}$ Recently, a randomized controlled trial - the Machine Preservation Trial - demonstrated that machine perfusion reduces the risk and duration of DGF compared to static cold storage in kidneys from deceased donors. ${ }^{17}$ However, this trial included a majority of DBD donors (87.5\%) and was not designed to allow detailed analysis of the effect of machine perfusion on DCD kidneys alone, thereby leaving this critical issue unresolved. 
Given this persisting controversy, we conducted a prospectively planned study as a prespecified extension of the Machine Preservation Trial to specifically determine the effect of machine perfusion versus static cold storage on post-transplant outcome of kidneys donated after cardiac death.

\section{Materials and Methods}

\section{$\underline{\text { Trial enrolment criteria }}$}

This prospectively planned analysis assessed all consecutive DCD kidney donors reported in Belgium and the Netherlands during the conduct of the Machine Preservation Trial. The study was fully integrated in the Eurotransplant system that manages waiting lists and organizes organ allocation in a part of Western Europe. ${ }^{18}$ The trial only included Maastricht category III (cardiac arrest after withdrawal of treatment) DCD donors aged 16 years or older. ${ }^{8}$ A strictly paired design was maintained, in which both kidneys of one donor needed to be transplanted into different recipients. Both kidneys of a pair were excluded from analysis when one or both recipients died within one week after transplantation. To allow complete integration within Eurotransplant, to reflect current practice and to assure the participation of all transplant centers, current standard center protocols were not changed. Informed consent from recipients was not required, as kidneys were randomized before organ allocation. Ethical approval was obtained from the Eurotransplant Ethical Advisory Committee, the Kidney Advisory Committee, and ethics review boards in each trial region.

\section{$\underline{\text { Randomization }}$}

Whenever a potential kidney donor meeting the inclusion criteria was reported, the Eurotransplant duty desk officer randomly assigned one kidney to machine perfusion and the contralateral kidney to static cold storage. Randomization lists were computer generated by the permuted block method. We used regional lists to avoid imbalances caused by small differences in allocation algorithms. When a reliable connection to the perfusion machine was impaired by a too small aortic patch or too many renal arteries, randomization for this kidney pair was changed and preservation methods were 
switched. Kidneys were allocated according to standard Eurotransplant allocation rules without revealing the preservation method at organ offer. The recipient's surgical team was unblinded at the time of transplantation.

\section{Preservation methods}

Hypothermic machine perfusion was performed with LifePort Kidney Transporter machines (Organ Recovery Systems, Itasca, IL, USA). For the purpose of the study, a trained perfusionist attended each donor procedure in order to guarantee availability and correct use of the machines. Immediately after organ recovery, the donor surgeon, assisted by the perfusionist, connected the kidney randomized to machine perfusion to the perfusion machine. A pulsatile flow with Kidney Preservation Solution-1 (KPS-1) $\left(1-8^{\circ} \mathrm{C}\right)$ was maintained until transplantation. ${ }^{19}$ Systolic perfusion pressure was fixed at $30 \mathrm{mmHg}$. Next, the machine-perfused kidney was transported to the recipient hospital without any monitoring. Flow readings and intravascular resistance were concealed to the transplantation team. As a result, the decision to accept or reject a kidney could not be biased by these parameters.

Kidneys randomized to static cold storage were flushed and preserved according to the established Eurotransplant routine, using either University of Wisconsin solution (UW) or histidine-tryptophanketoglutarate (HTK) according to center specific practice. Organs were submerged in the preservation solution and stored on melting ice until transplantation.

\section{Follow up}

No changes to center specific patient follow up protocols were made. Eurotransplant established a secure online database where follow up data could be provided by participating transplantation centers. To ensure maximal data completeness, recipient centers were financially compensated for providing follow up data. No relevant irregularities were found during an external audit of a random sample of $10 \%$ of all patient follow up data.

\section{$\underline{\text { Study endpoints }}$}


The primary endpoint was DGF, defined as the need for dialysis in the first week after transplantation. As a secondary endpoint, early graft function was assessed in a more refined qualitative way as functional DGF. Functional DGF was defined as the absence of a decrease in serum creatinine level by a minimum of $10 \%$ per day during three consecutive days in the first postoperative week, not including patients in whom acute rejection, calcineurin inhibitor toxicity, or both developed within the first week. ${ }^{20}$ Other secondary endpoints were: duration of DGF, primary non-function (PNF), biopsy-proven acute rejection, calcineurin inhibitor toxicity, serum creatinine values, creatinine clearance, length of recipients' hospital stay, and patient and graft survival up to one year after transplantation. Data on graft survival were censored at the time of death in patients who died with a functioning graft.

\section{$\underline{\text { Statistical methods }}$}

All data analyses were performed using SPSS, SAS, and R software. Two-sided p-values equal to or lower than 0.05 were considered to indicate statistical significance. The study was powered to detect a reduction in DGF due to machine perfusion of at least $20 \%$, based on a presumed rate of DGF of $70 \%$ in the cold storage arm. A minimum of 80 kidney pairs were required to obtain a statistical power of 0.8 , assuming a univariate one-sided type I error of 0.05 ; this is equivalent to the required sample size for a multivariate logistic regression with a two-sided type I error and similar power. ${ }^{21}$ The influence of machine perfusion compared to static cold storage on the risk of DGF was examined by a logistic regression model. ${ }^{21}$ Covariate selection was based on relevant literature and prespecified in the protocol before the trial started. ${ }^{22,}{ }^{23}$ To better reflect the paired study design, all covariates were entered in the analysis with a built-in normal gamma frailty term for the donor. Demographic variables were analyzed by Fisher's exact test or Mann-Whitney test. We applied McNemar's test or Wilcoxon signed-rank test to evaluate univariate differences in endpoint variables between the two groups. Assessment of graft and patient survival was done by the Kaplan-Meier method and differences between survival curves were determined by log-rank tests. Endpoint 
interim analyses were not performed, but confidential safety analyses comparing reported rates of adverse events in the two study arms were conducted at regular intervals by the trial safety board.

\section{$\underline{\text { Role of funding source }}$}

An independent scientific steering committee composed of clinicians and scientists from each trial region was responsible for the design, conduct, data analysis, and manuscript preparation for this study. The sponsor was not involved in the study design, follow up data acquisition, data analyses, or writing of the manuscript. During the course of this trial, the sponsor provided the trial regions with machine-perfusion devices and disposables free of charge and operated a 24-hour helpline that could be consulted by perfusionists in case of perfusion device-related technical issues.

\section{Results}

Kidney pairs donated after cardiac death were enrolled into the present study in two phases. In the first phase (1 November 2005 - 31 October 2006), enrolment was conducted as part of the larger Machine Preservation Trial. ${ }^{17}$ Near the end of donor enrolment in this main trial, the steering committee anticipated that insufficient DCD kidney pairs would be included to perform relevant analyses for the pre-specified DCD subgroup. Inclusion of DCD donors therefore was continued in a second phase (1 November 2006 - 17 August 2007) following the identical protocol of the Machine Preservation Trial. The flow diagram (Figure 1) shows enrolment and randomization of kidney pairs for the present study. Two hundred and four potential DCD kidney donors were assessed for inclusion, 103 kidney pairs were enrolled, and data from 82 recipients in each study group were analyzed. In nine cases, the connection of the kidney randomized to machine perfusion was unreliable because of aberrant vascular anatomy, and therefore preservation methods of both kidneys were switched. Vascular anomalies however did not significantly increase the risk of DGF (data not shown, $\mathrm{p}=0.064$ ).

\section{Study group characteristics}

Table 1 shows the characteristics of kidney donors and recipients. Eighty-two recipients were included in each study arm. There were no significant differences between the groups with respect 
to donor and recipient age, duration of pre-transplant dialysis, number of previous transplants, panel reactive antibodies, cold ischemic time, flush solution, induction therapy and maintenance immunosuppression regimens.

\section{Primary endpoint}

Forty-four recipients in the machine perfusion group and 57 recipients in the static cold storage group developed DGF (53.7\% vs. 69.5\%; $p=0.007$ ) (Table 2). Multivariate analysis (Table 3 ) showed a decreased probability of developing DGF in machine-perfused versus static cold-stored DCD kidneys (adjusted odds ratio $0.43 ; 95 \%$ confidence interval $0.20-0.89 ; p=0.025$ ). Other significant risk factors for DGF were donor and recipient age and warm and cold ischemic time.

\section{Secondary endpoints}

Table 2 shows the univariate analysis of all secondary endpoints. Functional DGF occurred in 16 recipients in the machine perfusion group versus 42 recipients in the static cold storage group (19.5\% vs. $51.2 \% ; \mathrm{p}<0.0001)$. The median duration of DGF in the machine perfusion group was four days shorter than in the static cold storage group, but this difference did not reach statistical significance (9 vs. 13 days; $p=0.082$ ). However, DGF was more likely to be shorter than seven days in a machineperfused kidney compared with a static cold-stored kidney. There were no differences in the median length of recipients' hospital stay. PNF occurred in only two cases in each study group. Creatinine clearance was significantly higher in the machine perfusion group until one month after transplantation. At one year follow up, three patients in the machine perfusion group and two patients in the static cold storage group had died. Graft survival at one year follow up was similar in both groups (93.9\% vs. 95.1\%) (Figure 2).

\section{Complications}

No vascular complications of the graft (arterial thrombosis, dissection ... ) were seen in either group. Cardiovascular, gastro-intestinal, infectious, metabolic, urinary and technical complications were comparable between the groups and within reported ranges in the literature (data not shown). 


\section{Discussion}

This multicenter randomized, controlled trial demonstrated the superiority of machine perfusion over static cold storage for the preservation of DCD kidneys. This is an important finding, as DGF after kidney transplantation adversely influences outcome, causes morbidity and even mortality in older recipients, and leads to additional costs. ${ }^{1-6}$ DCD kidneys are currently accepted by many transplant centers as an additional donor source and the potential of DCD kidneys is large. As DCD kidneys are intrinsically more prone to developing DGF, decreasing the incidence of DGF by machine perfusion will be particularly beneficial for recipients of this type of kidney graft. ${ }^{8-10}$

In the multivariate analysis, machine perfusion clearly reduced the risk of DGF. Furthermore, DGF was more likely to be short lasting (less than seven days) in machine-perfused kidneys compared with static cold-stored kidneys. We also explored the impact of machine perfusion on functional DGF. Compared to DGF defined as dialysis requirement in the first postoperative week, functional DGF is a more refined surrogate marker for early kidney graft function. ${ }^{20}$ We found that the incidence of functional DGF was strongly reduced by machine perfusion, even more than the incidence of DGF. Hence, the protective effect of machine perfusion shown in our study may be underestimated when using only the traditional definition of DGF as an outcome measure. However, we selected the traditional definition of DGF as the primary endpoint to allow for comparison of the results in the present analysis with those from previous studies. Our observation that creatinine clearance in recipients of machine-perfused kidneys was higher early after transplantation shows that actual early kidney function is also superior after machine perfusion.

Our study confirmed that donor age and cold ischemic time are independent risk factors for DGF in DCD kidneys, even though cold ischemic times were relatively short in both groups. ${ }^{22,}{ }^{23}$ Cold ischemic time was slightly but not significantly longer in the static cold stored group. However, with a previously reported odds ratio of 1.23 of DGF for every six hour increase in cold ischemic time ${ }^{24}$, it is unlikely that these additional 54 minutes of cold ischemia caused a major bias of the primary 
endpoint. Moreover, the study revealed that the duration of warm ischemia is a more important independent additional risk factor for DGF.

Even though DGF is a risk factor for graft failure after kidney transplantation and machine perfusion significantly reduced the risk for DGF, our study did not show improvement in one-year graft survival of machine-perfused versus static cold-stored kidneys. ${ }^{1-3}$ We cannot exclude that the young donor age in our cohort in part masked an advantage of machine perfusion on graft survival. Nevertheless, this finding is in line with an increasing number of reports showing similar mediumterm graft survival for DCD and DBD kidneys despite higher rates of DGF in DCD kidneys. ${ }^{8,10,25}$ DGF does not influence graft survival after DCD kidney transplantation in the same way it does after DBD kidney transplantation. This could be explained by a possibly different nature of DGF in DCD versus DBD kidneys. The metabolic, hemodynamic, hormonal, and inflammatory changes that occur after brain death and during donor management, but not after cardiac death, may impair kidney function more and could have more long-term impact than warm ischemic injury alone. ${ }^{26,27}$

The present study yielded a few surprising results. First, despite the significant reduction of DGF by machine perfusion, the incidence of PNF was not reduced. A PNF incidence of only $2.4 \%$ may appear surprisingly low. The exclusion of uncontrolled (Maastricht category I and II) DCD donors that are more prone to PNF and the relatively short median warm and cold ischemic times in our donor population may account for the low rate of PNF. However, when compared to reported PNF incidences in other series of controlled DCD kidney transplantations (0\%-17\%) and the previously conducted main trial (1/42), the observed incidence of PNF was not exceptionally low. ${ }^{11,12,14,17,28,29}$ Nevertheless, it is likely that the overall incidence of PNF was too low to detect an effect of machine perfusion. Second, hospital stay is usually longer in recipients of DCD versus DBD kidneys because of the increased rate of DGF in the former group. ${ }^{10}$ Despite reduced duration and severity of DGF, our study showed no significant reduction in hospital stay for recipients of machine-perfused kidneys. This unexpected observation may, at least in part, be explained by the fact that the trial was conducted in Eurotransplant countries. Healthcare systems with greater pressure to limit the use of 
resources will have a tendency towards shorter hospital stay. ${ }^{30,31}$ We believe that in countries with such a healthcare system, the reduction in DGF seen in our trial might be paralleled by a significant reduction in hospital stay. This observation also reflects the relative unreliability of hospital stay as a valid outcome parameter, as suggested by other studies.

To date, no definitive evidence of the superiority of machine perfusion over cold storage for the preservation of DCD kidneys has been available. Although an advantage of machine perfusion has been suggested, all previous studies were relatively small in size compared to the present prospective trial. ${ }^{11-13,16}$ The effect of machine perfusion on DCD kidney preservation was recently studied in the United Kingdom. A randomized controlled trial with sequential analysis suggested that machine perfusion of DCD kidneys does not decrease DGF. ${ }^{29}$ To our knowledge, this trial is so far unpublished. Differences in study design may account for this discrepancy. The present trial was not only larger but was also fully integrated into Eurotransplant. Kidneys were allocated strictly and solely according to standard Eurotransplant rules, and recipient centers were blinded to the preservation method at the time of organ offer. Furthermore, all kidneys were perfused immediately after retrieval until transplantation, which was not necessarily the case in other studies. The need to perfuse kidneys immediately after retrieval to benefit fully from the "perfusion effect" needs to be investigated further, as this practice has important logistic consequences.

The present study has some limitations. The strictly paired design of the trial and the necessity to randomize kidney pairs immediately after the report of a potential donor, accounts for the large number of exclusions. First, donor kidney pairs of which one kidney was not transplanted were excluded, possibly leaving kidneys with a higher risk of DGF out of the study. Second, less hemodynamically stable donors in whom organ recovery had to be performed as an emergency procedure could not always be reached in time. Another possible limitation is the difference in preservation solutions in both groups. Only one pharmaceutical formulation of machine preservation solution is FDA approved, therefore machine-perfused kidneys were preserved with KPS-1. Static cold-stored kidneys were preserved in HTK (75.6\%) or UW $(22.0 \%)$ and although UW is publisher's copy-editing and typesetting process, which will usually result in changes to the font and text alignment. 
still the gold standard for cold storage of kidneys, analysis of the United Network of Organ Sharing data showed that HTK preservation has no effect on DGF compared to UW. ${ }^{32}$

In conclusion, this international randomized, controlled trial showed that hypothermic machine perfusion of DCD kidneys reduced the risk of DGF and improved graft function until one month after transplantation. When DGF occurred, it was of a shorter duration and less severe. We therefore suggest that machine perfusion should be routinely used for the preservation of DCD kidneys. Apart from being beneficial to the individual patient, these protective effects of machine perfusion might result in a substantial cost reduction of DGF-related costs. The cost-effectiveness of hypothermic machine perfusion however remains to be investigated.

This is the version of the paper as accepted by "Ann Surg".

This version has been through peer-review but still contains the authors' original formatting. It has not yet undergone the publisher's copy-editing and typesetting process, which will usually result in changes to the font and text alignment. 


\section{Acknowledgments}

Organ Recovery Systems supported this trial.

IJ is a doctoral fellow associated with the Research Foundation-Flanders, Belgium.

CM receives grant support from the Dutch Kidney Foundation.

JP and DM received grant support from the government of Flanders, Belgium, in cooperation with

Organ Recovery Systems to study machine perfusion of liver grafts, for which they receive no salary.

$\mathrm{CM}, \mathrm{AP}, \mathrm{HGDL}$, JPS, and JP received congress travel grants from Organ Recovery Systems.

RJP receives consultancy fees from Bristol-Myers Squibb and grant support from the Dutch Kidney Foundation and the Eurotrans-Bio pro-donor project.

RJP and HGDL are listed as inventors in a patent on a portable preservation apparatus for donor organs. 


\section{References}

1. Perico N, Cattaneo D, Sayegh $\mathrm{MH}$, et al. Delayed graft function in kidney transplantation. Lancet 2004; 364:1814-27.

2. Yarlagadda SG, Coca SG, Formica RN, Jr., et al. Association between delayed graft function and allograft and patient survival: a systematic review and meta-analysis. Nephrol Dial Transplant 2009; 24:1039-47.

3. Halloran PF, Hunsicker LG. Delayed graft function: state of the art, November 10-11, 2000. Summit meeting, Scottsdale, Arizona, USA. Am J Transplant 2001; 1:115-20.

4. Kauffman HM, McBride MA, Cors CS, et al. Early mortality rates in older kidney recipients with comorbid risk factors. Transplantation 2007; 83:404-10.

5. Rosenthal JT, Danovitch GM, Wilkinson A, et al. The high cost of delayed graft function in cadaveric renal transplantation. Transplantation 1991; 51:1115-8.

6. Hagenmeyer EG, Haussler B, Hempel E, et al. Resource use and treatment costs after kidney transplantation: impact of demographic factors, comorbidities, and complications. Transplantation 2004; 77:1545-50.

7. Daemen JW, Oomen AP, Kelders WP, et al. The potential pool of non-heart-beating kidney donors. Clin Transplant 1997; 11:149-54.

8. Kootstra G, van Heurn E. Non-heartbeating donation of kidneys for transplantation. Nat Clin Pract Nephrol 2007; 3:154-63.

9. Moers C, Leuvenink HG, Ploeg RJ. Non-heart beating organ donation: overview and future perspectives. Transpl Int 2007; 20:567-75.

10. Kokkinos C, Antcliffe D, Nanidis T, et al. Outcome of kidney transplantation from nonheartbeating versus heart-beating cadaveric donors. Transplantation 2007; 83:1193-9.

11. Plata-Munoz JJ, Muthusamy A, Quiroga I, et al. Impact of pulsatile perfusion on postoperative outcome of kidneys from controlled donors after cardiac death. Transpl Int 2008; 21:899907. 
12. Moustafellos $\mathrm{P}$, Hadjianastassiou $\mathrm{V}$, Roy $\mathrm{D}$, et al. The influence of pulsatile preservation in kidney transplantation from non-heart-beating donors. Transplant Proc 2007; 39:1323-5.

13. St Peter SD, Imber CJ, Friend PJ. Liver and kidney preservation by perfusion. Lancet 2002; 359:604-13.

14. van der Vliet JA, Kievit JK, Hene RJ, et al. Preservation of non-heart-beating donor kidneys: a clinical prospective randomised case-control study of machine perfusion versus cold storage. Transplant Proc 2001; 33:847.

15. Opelz G, Terasaki PI. Advantage of cold storage over machine perfusion for preservation of cadaver kidneys. Transplantation 1982; 33:64-8.

16. Wight J, Chilcott J, Holmes M, et al. The clinical and cost-effectiveness of pulsatile machine perfusion versus cold storage of kidneys for transplantation retrieved from heart-beating and non-heart-beating donors. Health Technol Assess 2003; 7:1-94.

17. Moers C, Smits JM, Maathuis MH, et al. Machine Perfusion or Cold Storage in DeceasedDonor Kidney Transplantation. N Engl J Med 2009; 360:7-19.

18. Eurotransplant. http://www.eurotransplant.nl/. Accessed August 19, 2009.

19. Belzer FO, Glass NR, Sollinger HW, et al. A new perfusate for kidney preservation. Transplantation 1982; 33:322-3.

20. Boom H, Paul L, de Fijter J. Delayed graft function in renal transplantation. Transplant Rev 2004; 18:139-152.

21. Hsieh FY, Bloch DA, Larsen MD. A simple method of sample size calculation for linear and logistic regression. Stat Med 1998; 17:1623-34.

22. McLaren AJ, Jassem W, Gray DW, et al. Delayed graft function: risk factors and the relative effects of early function and acute rejection on long-term survival in cadaveric renal transplantation. Clin Transplant 1999; 13:266-72. 
23. Troppmann C, Gillingham KJ, Benedetti E, et al. Delayed graft function, acute rejection, and outcome after cadaver renal transplantation. The multivariate analysis. Transplantation $1995 ; 59: 962-8$.

24. Ojo AO, Wolfe RA, Held PJ, et al. Delayed graft function: risk factors and implications for renal allograft survival. Transplantation 1997; 63:968-74.

25. Brook NR, Waller JR, Nicholson ML. Nonheart-beating kidney donation: current practice and future developments. Kidney Int 2003; 63:1516-29.

26. Rowinski W, Walaszewski J, Lagiewska B, et al. Use of kidneys from marginal and non-heartbeating donors: warm ischemia per se is not the most detrimental factor. Transplant Proc 1993; 25:1511-2.

27. Barklin A. Systemic inflammation in the brain-dead organ donor. Acta Anaesthesiol Scand $2009 ; 53: 425-35$.

28. Daemen JH, de Vries B, Kootstra G. The effect of machine perfusion preservation on early function of non-heart-beating donor kidneys. Transplant Proc 1997; 29:3489.

29. Watson C, Wells A, Roberts R, et al. Machine Perfusion of Kidneys donated after Cardiac Death does not reduce Delayed Graft Function. Am J Transplant 2008; 8:224.

30. Clarke A, Rosen R. Length of stay. How short should hospital care be? Eur J Public Health $2001 ; 11: 166-70$.

31. Morgan M, Beech R. Variations in lengths of stay and rates of day case surgery: implications for the efficiency of surgical management. J Epidemiol Community Health 1990; 44:90-105.

32. Stewart ZA, Lonze BE, Warren DS, et al. Histidine-tryptophan-ketoglutarate (HTK) is associated with reduced graft survival of deceased donor kidney transplants. Am J Transplant 2009; 9:1048-54.

33. Metzger RA, Delmonico FL, Feng S, et al. Expanded criteria donors for kidney transplantation. Am J Transplant 2003; 3:114-25. 
Tables and Figures

Table 1. Characteristics of donors and recipients.

\begin{tabular}{|c|c|c|c|}
\hline Variable & $\begin{array}{l}\text { Machine perfusion } \\
\text { group }(n=82)\end{array}$ & $\begin{array}{l}\text { Static cold storage } \\
\text { group }(n=82)\end{array}$ & $p$-value \\
\hline \multicolumn{4}{|l|}{ Donor characteristics } \\
\hline$\overline{\operatorname{Age}^{a, b}(y r)}$ & \multicolumn{2}{|c|}{$43(17-67)$} & \\
\hline Warm ischemic time ${ }^{c}(\min )$ & \multicolumn{2}{|c|}{$16(6-38)$} & \\
\hline$<10(\mathrm{n})$ & \multicolumn{2}{|c|}{21} & \\
\hline $10-19(n)$ & \multicolumn{2}{|c|}{40} & \\
\hline $20-29(n)$ & \multicolumn{2}{|c|}{18} & \\
\hline$\geq 30(n)$ & \multirow{2}{*}{\multicolumn{2}{|c|}{$\begin{array}{c}6 \\
62 / 18 / 2\end{array}$}} & \\
\hline Flush solution: HTK/UW/other & & & \\
\hline \multicolumn{4}{|l|}{ Cold ischemic time $^{\mathrm{d}}$} \\
\hline Median (range) & $15.0(4.3-28.9)$ & $15.9(8.6-46.6)$ & 0.70 \\
\hline Mean (25-75 percentile) & $16.6(14.2-19.8)$ & $17.3(13.9-19.7)$ & 0.41 \\
\hline$>24 \mathrm{hr}(\mathrm{n})$ & 4 & 6 & \\
\hline \multicolumn{4}{|l|}{ Recipient characteristics } \\
\hline$\overline{\operatorname{Age}^{a}(y r)}$ & $49(24-73)$ & $52(24-77)$ & 0.81 \\
\hline Duration pre-transplant dialysis ${ }^{a}(y r)$ & $4.2(1.0-17.5)$ & $4.0(0.4-10.7)$ & 0.48 \\
\hline Previous transplants $(n)$ & & & 0.82 \\
\hline First transplant & 71 & 70 & \\
\hline Re-transplant & 11 & 12 & \\
\hline Panel reactive antibodies (\%) & & & 0.73 \\
\hline $0-5(n)$ & 71 & 71 & \\
\hline $6-84(n)$ & 11 & 10 & \\
\hline$\geq 85(n)$ & 0 & 1 & \\
\hline $\begin{array}{l}\text { No mismatches at HLA-A, B, DR loci (9 } \\
\text { Immunosuppression (n) }\end{array}$ & 2.4 & 3.7 & 0.50 \\
\hline Anti-thymocyte globulin & 12 & 13 & 0.71 \\
\hline Interleukin 2 receptor antagonist & 37 & 31 & 0.34 \\
\hline Azathioprine & 1 & 1 & 0.61 \\
\hline Cyclosporin A & 37 & 31 & 0.34 \\
\hline Tacrolimus & 43 & 52 & 0.25 \\
\hline Corticosteroids & 81 & 81 & 1.00 \\
\hline Mycophenolate mofetil & 69 & 76 & 0.14 \\
\hline
\end{tabular}

${ }^{\text {a }}$ Median (range)

b 14 DCD donors also fulfilled the criteria for expanded criteria donors as determined by the United Network for Organ Sharing, 8 were older than 60 years of age. ${ }^{33}$

${ }^{c}$ Warm ischemic time: time from circulatory arrest until the start of cold perfusion.

${ }^{d}$ Cold ischemic time: time from start cold perfusion until the start of kidney implantation. 
Table 2. Univariate analysis of trial endpoints.

\begin{tabular}{|c|c|c|c|}
\hline Variable & $\begin{array}{l}\text { Machine perfusion } \\
\text { group }(n=82)\end{array}$ & $\begin{array}{l}\text { Static cold storage } \\
\text { group }(n=82)\end{array}$ & $p$-value \\
\hline \multicolumn{4}{|l|}{ Delayed graft function ${ }^{a, b}$} \\
\hline Incidence $(\mathrm{n})$ & $44(53.7 \%)$ & $57(69.5 \%)$ & 0.007 \\
\hline Duration & & & 0.021 \\
\hline$<7$ days & 12 & 6 & \\
\hline$\geq 7$ days & 32 & 51 & \\
\hline Median duration (days) & $9(1-48)$ & $13(2-43)$ & 0.082 \\
\hline Functional delayed graft function ${ }^{a, c}(n)$ & $16(19.5 \%)$ & $42(51.2 \%)$ & $<0.0001$ \\
\hline Primary non-function ${ }^{a, d}$ & $2(2.4 \%)$ & $2(2.4 \%)$ & 1.00 \\
\hline Acute rejection within 14 days ( $n$ ) & $6(7.3 \%)$ & $10(12.2 \%)$ & 0.28 \\
\hline Calcineurin inhibitor toxicity $(\mathrm{n})$ & $13(15.9 \%)$ & $10(12.2 \%)$ & 0.34 \\
\hline \multicolumn{4}{|l|}{ Serum creatinine value ${ }^{a}(\mathrm{mg} / \mathrm{dl})$} \\
\hline 14 days post-transplant & $4.1(0.9-11.2)$ & $5.1(1.0-11.3)$ & 0.001 \\
\hline 1 month post-transplant & $1.7(0.9-7.1)$ & $2.1(0.7-9.9)$ & 0.017 \\
\hline 3 months post-transplant & $1.5(0.8-5.4)$ & $1.5(0.6-8.3)$ & 0.021 \\
\hline \multicolumn{4}{|l|}{ Creatinine clearance ${ }^{a}(\mathrm{ml} / \mathrm{min})$} \\
\hline 14 days post-transplant & $23(3-98)$ & $13(0-160)$ & $<0.0001$ \\
\hline 1 month post-transplant & $46(10-98)$ & $35(1-113)$ & 0.027 \\
\hline 3 months post-transplant & $57(11-128)$ & $49(11-104)$ & 0.117 \\
\hline Length of recipient hospital stay ${ }^{a}$ (days) & $17(7-392)$ & $19(8-65)$ & 0.24 \\
\hline \multicolumn{4}{|l|}{ Allograft survival } \\
\hline at 3 months & 79 (96.3\%) & 79 (96.3\%) & \\
\hline at 1 year & $77(93.9 \%)$ & $78(95.1 \%)$ & \\
\hline \multicolumn{4}{|l|}{ Recipient survival } \\
\hline at 3 months & $81(98.8 \%)$ & $82(100 \%)$ & \\
\hline at 1 year & $79(96.3 \%)$ & $80(97.6 \%)$ & \\
\hline
\end{tabular}

${ }^{a}$ Median (range).

${ }^{\mathrm{b}}$ Delayed graft function: need for dialysis in the first week after transplantation.

${ }^{\mathrm{C}}$ Functional delayed graft function: lack of $\geq 10 \%$ serum creatinine decrease per day during three consecutive days in the first week after transplantation. ${ }^{20}$ Recipients developing acute rejection or calcineurin inhibitor toxicity within the first week were excluded from this category.

${ }^{d}$ Primary non-function: permanent lack of graft function. 
Table 3. Multivariate analysis of the risk of delayed graft function

\begin{tabular}{lcc}
\hline Variable & $\begin{array}{c}\text { Adjusted odds ratio } \\
\text { (95\% confidence } \\
\text { interval) }\end{array}$ & p-value \\
\hline Machine perfusion vs. static cold storage & $0.43(0.20-0.89)$ & 0.025 \\
Donor age (yr) & $1.04(1.01-1.08)$ & 0.008 \\
Recipient age (yr) & $1.04(1.00-1.08)$ & 0.028 \\
Re-transplant vs. first transplant & $0.77(0.39-1.54)$ & 0.46 \\
Panel reactive antibody level (\%) & $2.97(0.90-9.87)$ & 0.075 \\
HLA mismatches (n) & $1.28(0.87-1.88)$ & 0.21 \\
Duration of pre-transplantation dialysis (days) & $1.01(0.88-1.27)$ & 0.92 \\
Cold ischemic time (h) & $1.10(1.01-1.21)$ & 0.039 \\
Warm ischemic time (10 min) & $3.40(1.87-6.17)$ & $<0.0001$ \\
\hline
\end{tabular}

${ }^{a}$ Warm ischemic time: time from circulatory arrest until the start of cold perfusion.

Warm ischemic time was grouped in 10 minute intervals and a warm ischemic time $<10$ min was used as baseline. 


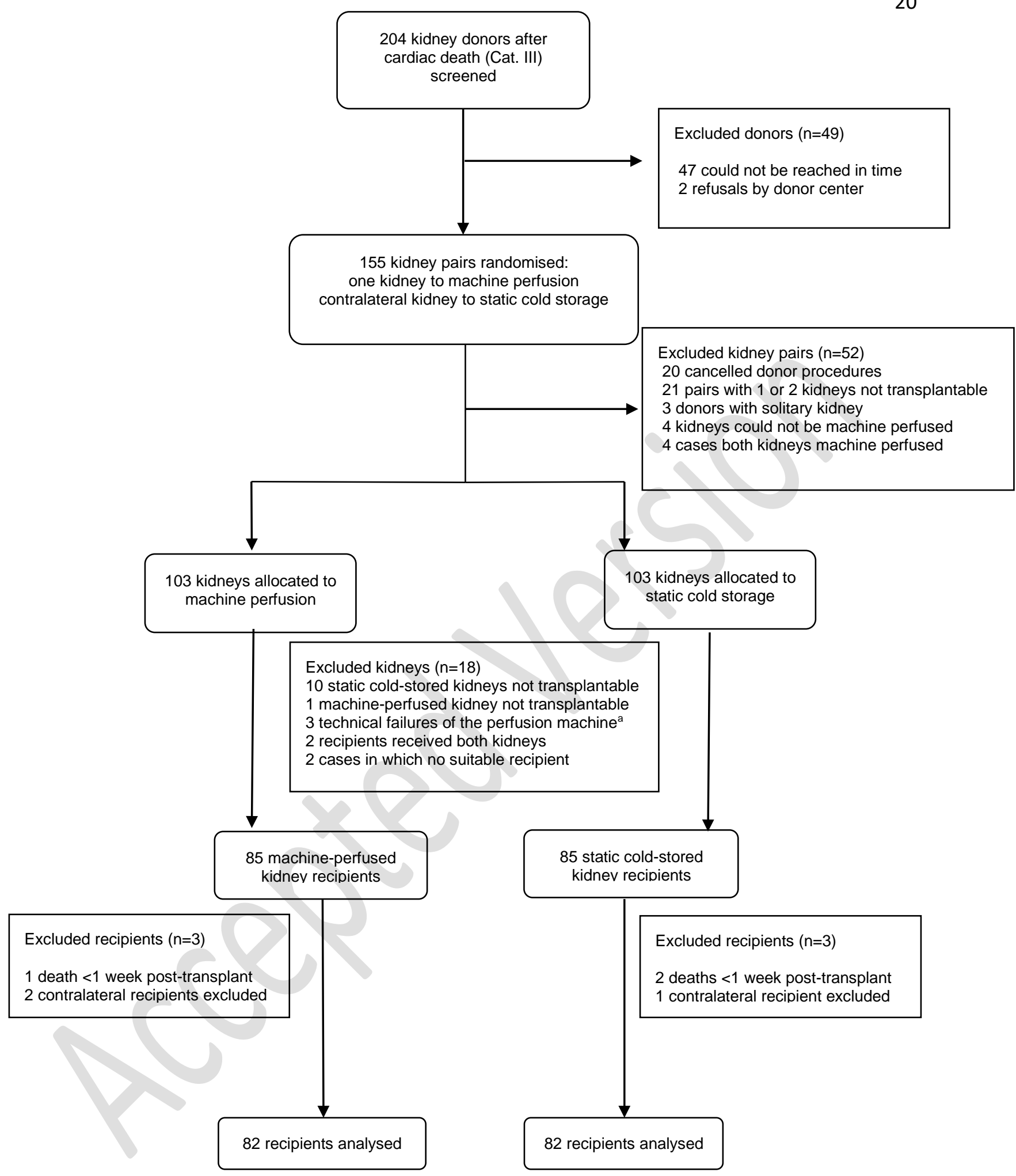

Figure 1. CONSORT diagram showing enrolment and randomization of kidney pairs in the trial

a Technical machine related problems caused the machine to switch to the "fail safe" mode and led to cold storage of the kidney inside the machine. These kidneys remained suitable for transplantation but were excluded from analysis in the present study.

Because the machine perfusion pump is pressure controlled, the "fail safe" mode is activated when a risk of possible barotrauma is detected. This occurred in three cases: (1) a sudden change in surrounding pressure during transport misguided the software, (2) a high resistance alarm and (3) a leakage of perfusion fluid.

This is the version of the paper as accepted by "Ann Surg".

This version has been through peer-review but still contains the authors' original formatting. It has not yet undergone the publisher's copy-editing and typesetting process, which will usually result in changes to the font and text alignment. 


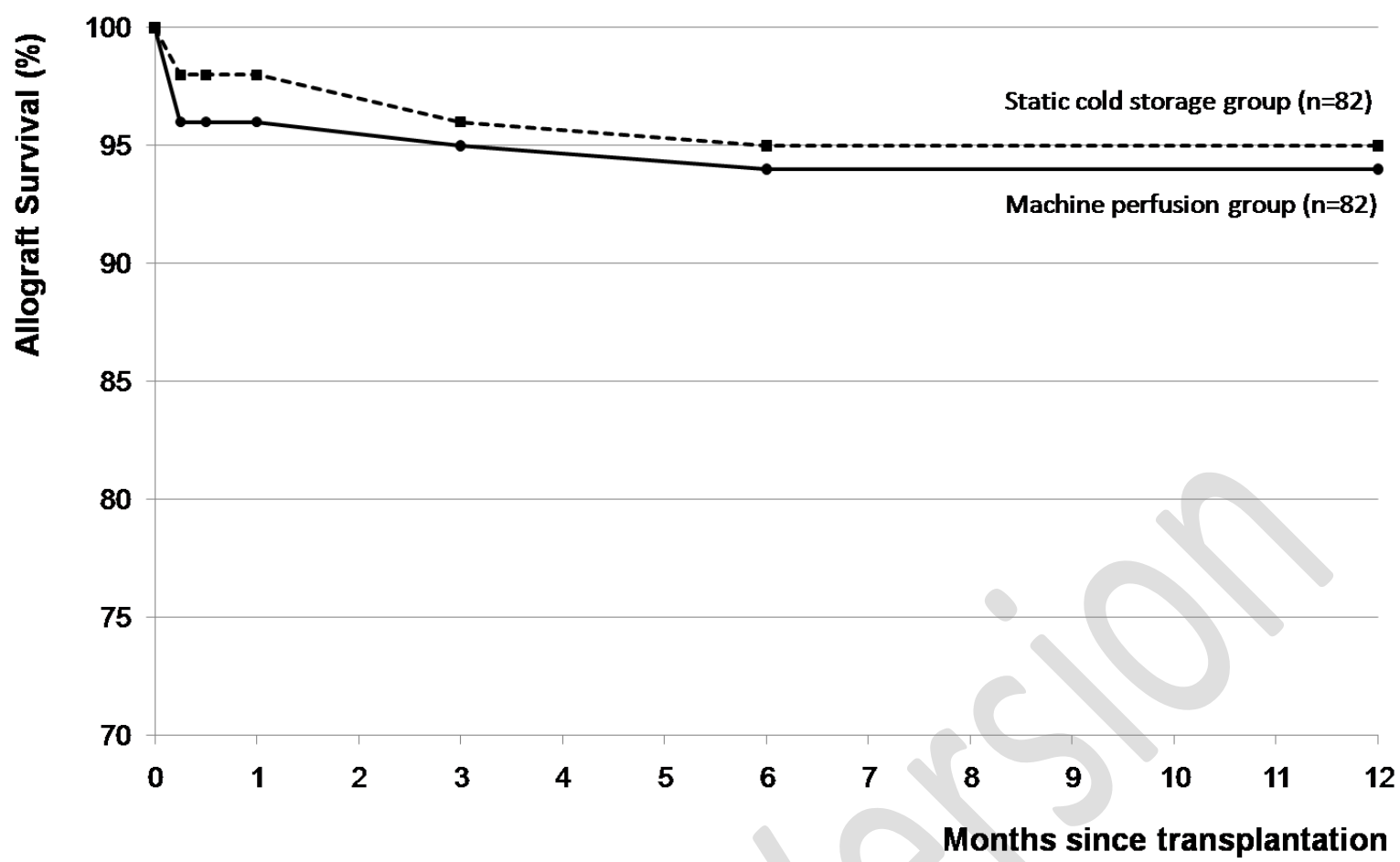

Figure 2. Death censored allograft survival at one year after transplantation.

Graft survival in the machine perfusion versus the static cold storage group was similar (94\% vs. 95\%) (Log-rank test of equality: $p=0.7$ ).

This is the version of the paper as accepted by "Ann Surg".

This version has been through peer-review but still contains the authors' original formatting. It has not yet undergone the publisher's copy-editing and typesetting process, which will usually result in changes to the font and text alignment. 